\title{
Defective and Repairable Items Unsteady Deterioration Rate Inventory Model Under Three Tired Prices and Time Dependent Demand D.M. Patel ${ }^{1 *}$, R.D. Patel $^{2}$
}

\footnotetext{
${ }^{1}$ Department of Commerce, Narmada College of Science and Commerce, Zadeshwar, Bharuch, India

${ }^{2}$ Visiting Professor, M. Sc. (IT) Program, Veer Narmad South Gujarat University, Surat, India
}

DOI: $10.36347 /$ sipms.2021.v08i05.002

| Received: 05.04.2021 | Accepted: 17.05.2021 | Published: 22.05.2021

*Corresponding author: D.M. Patel

Abstract

Lot received or units produced are not all perfect items. A time and price dependent demand inventory model is formulated when items produced or lot received are of defective and repairable nature. Three tired pricing is considered. For different situations, expression for total profit is derived to derive optimal solution. For parameter, post-optimality computations are also done.

Keywords: Inventory model, Varying deterioration, Price dependent demand, Time dependent demand, Three tire pricing, Defective items, Repairable items, Time varying holding cost.

Copyright $(\mathcal{C} 2021$ The Author(s): This is an open-access article distributed under the terms of the Creative Commons Attribution 4.0 International License (CC BY-NC 4.0) which permits unrestricted use, distribution, and reproduction in any medium for non-commercial use provided the original author and source are credited.

\section{INTRODUCTION}

Everywhere inventories are essential. Absence of inventory make customer uncomfortable and it may affect goodwill or profit of future of the organization. Also certain items are such that their value reduces during their storage period and we say these items as deteriorating items. In last few decays much attention has been given towards developing decaying items models. A stock model under fixed deterioration rate was proposed by Ghare and Schrader [1963]. Subsequently, taking into account changeable deterioration rate, model was expanded by Covert and Philip [1973]. Under selling price dependent demand and Weibull decay rate, Aggarwal and Goel [1984] obtained an inventory model. Mukhopadhyay et al. [2004] obtained a stock model for decaying items under price dependent demand. A stock with selling price related inventory model for decaying units was formulated by Teng and Chang [2005]. Under changeable storage cost and stock dependent demand, a stock model was constructed by Alfares [2007]. A time and selling price dependent inventory model was obtained by Mathew [2013]. A stock level model under stock size and price fluctuating demand with varying deterioration was constructed by Patel and Sheikh [2015]. Under trade credit situation for noninstantaneous deteriorating items, a stock level model was formulated by Tsao et al. [2017].

Imperfect quality of goods affects management of inventory. Therefore this characteristic is to be taken into consideration. Several academicians studied and analysed difficulties associated with defective production process of an item. Lee and Rosenblatt [1985] proposed an imperfect quality items stock model for obtaining optimal order policy. An EOQ model that contains known proportion of defective units in received lot and to remove these items costs incurs of fixed and variable inspection nature was obtained by Schwaller [1988]. When production process is not perfect, an inventory model was formulated by Cheng [1991]. It was assumed that demand of item depends on unit production cost. An inventory model when received items are not $100 \%$ perfect was obtained by Salameh and Jaber [2000]. Imperfect units are separated after $100 \%$ screening and their selling will be done at discounted price. An EPQ model under known proportion of defective units in lot produced following a uniform distribution was obtained by Hayek and Salameh [2001]. A defective items production inventory model was obtained by Goyal and Barron [2002]. A no shortage defective items inventory model was constructed by Papachristos and Konstantaras [2006] in which at cycle end defective units has been removed. Under effect of learning, an EPQ model having defective units was obtained by Jaber et al. [2008]. Under stock out situation, Hsu and Hsu [2012] obtained a defective units stock model. An EPQ with rework to determine selling price, stock size and shipment size collectively was formulated by Taleizadeh et al. [2015]. A deteriorating items production inventory model for defective items was 
D.M. Patel \& R.D. Patel., Sch J Phys Math Stat, May, 2021; 8(5): 96-104

considered by Shukla et al. [2016]. Naik and Patel [2017] constructed a time and price related stock model for defective units under unsteady deterioration.

The term repairing/remanufacturing in inventory modelling was first introduced by Schrady [1967]. By considering multi-item system sharing the same repair facility and stock-out service level constraints, Mabini et al. [1992] expanded the model. A linear demand inventory model for defective items in which some items can be repairable, was considered by Yadav and Kumar [2014]. Gothi et al. [2017] formulated a linear demand and exponential type deterioration of items inventory model in which received items having defects but some of them can be repairable. Naik and Patel [2018] constructed a price and time dependent demand stock model for defective and repairable items under unsteady deterioration.

A varying deterioration inventory model for defective and repairable items is developed. Three tire pricing policy is adopted. Three tire price and time related demand function is considered. Stock outs are not permitted. Model is justified with numerical example and post-optimality computations.

\section{ASSUMPTIONS AND NOTATIONS NOTATIONS}

Notations used in modelare

$\mathrm{D}\left(\mathrm{p}_{\mathrm{i}}, \mathrm{t}\right)$ : Price and time dependent demand $\left(\mathrm{a}_{\mathrm{i}}+\mathrm{b}_{\mathrm{i}} \mathrm{t}-\rho_{\mathrm{i}} \mathrm{p}_{\mathrm{i}}\right.$, a $>0,0<b_{i}<1, \rho_{i}>0, i=1,2,3$ in different intervals)

c : Unit cost of purchasing of item

$\mathrm{p}_{\mathrm{i}} \quad$ : Selling price per unit of item in different intervals in a cycle

d : Imperfect articles (\%)

$1-\mathrm{d}: \%$ of perfect articles

$\mathrm{d}_{1} \quad$ : Items for repairing (in \%)

d :\% of perfect articles

$\mathrm{d}_{1} \quad$ : Items for repairing (in \%)

$\lambda \quad$ : Rate of screening

SR : Revenue from sales

A : Per order cost of replenishment

z : Screening cost of one item

$\mathrm{p}_{\mathrm{d}} \quad$ : Selling value of imperfect quality units

$h(t):$ Storage cost of item $(x+y t, x>0,0<y<1)$

$\mathrm{m}$ : Per unit cost of transportation of repairable items

$\mu_{1} \quad$ : Period of screening

$\mathrm{T}$ : Inventory cycle time

$\mathrm{I}(\mathrm{t})$ : At time $\mathrm{t}$, inventory size

Q : Quantity required in a cycle

$\theta \quad$ : Rate of deterioration in $\mathrm{t}_{1} \leq \mathrm{t} \leq \mathrm{t}_{2}, 0<\theta<1$

$\theta \mathrm{t} \quad$ : Rate of deterioration in $\mathrm{t}_{2} \leq \mathrm{t} \leq \mathrm{T}, 0<\theta<1$

$\pi \quad$ : Items per unit profit.

\section{ASSUMPTIONS}

Model is based on assumptions

- Demand of item is function of time and prices.
- Product has infinite and instantaneous replenishment rate.

- There is zero lead time.

- There are no shortages of items.

- During screening process, demand occurs but is less than screening rate $(\lambda)$ i.e. $\left(a_{i}+b_{i} t-\rho_{i} p_{i}\right)<\lambda$.

- Deterioration of items and defective items are independent.

- $\quad$ Some defective items are repairable items.

- In each cycle, no repairing or replacement of deteriorated items.

- Only one item is taken for analysis.

- Varying holding cost is considered.

- Screening machine takes very less time for inspection of items for verification means we say that screening rate $(\lambda)$ is sufficiently large.

\section{THE MATHEMATICAL MODEL AND ANALYSIS}

Items of amount $\mathrm{Q}$ are received at the starting of cycle. Out of Q units d\% of items are defective items and out of these defective items, repairable items are $\mathrm{d}_{1} \%$. These units, at rate of $\lambda$ per unit time as shown in figure below go through screening process during time 0 to $\mu_{1}$. Items which are found to be perfect are separated and demand occurred during 0 to $\mu_{1}$ will be fulfilled from these perfect quality items. Moreover from these defective items repairable items are separated and sent for repairing to manufacturer and remaining non-repairable units are sold at end of cycle at reduced price as a single batch. One cycle time is divided as $\left(0, t_{1}\right),\left(t_{1}, t_{2}\right)$ and $\left(t_{2}, T\right)$. In period $\left(0, t_{1}\right)$ there is no deterioration and price is $\mathrm{p}_{1}$, in period $\left(\mathrm{t}_{1}, \mathrm{t}_{2}\right)$ deterioration rate is $\theta$ and price is $\mathrm{p}_{2}$, in period $\left(\mathrm{t}_{2}, \mathrm{~T}\right)$ deterioration rate is $\theta t$ and price is $p_{3}$, (where $p_{1}>p_{2}>$ $\mathrm{p}_{3}$ ). At end of a cycle because of deterioration and demand, level of inventory reaches to zero.

Here $\mu_{1}=\frac{Q}{\lambda}$

And we put restriction for defective percentage (d) as:

$\mathrm{d} \leq 1-\frac{\left(\mathrm{a}_{\mathrm{i}}+\mathrm{b}_{\mathrm{i}} \mathrm{t}-\rho_{\mathrm{i}} \mathrm{p}_{\mathrm{i}}\right)}{\lambda}$.

During cycle time $(0 \leq \mathrm{t} \leq \mathrm{T})$ inventory size is as shown below:

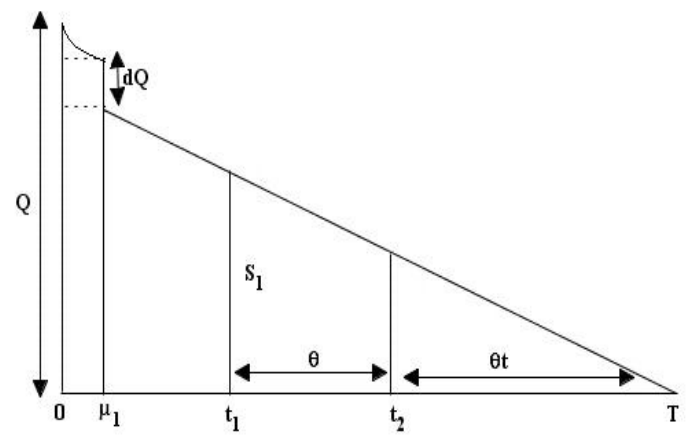

Fig-1 
For period $(0, \mathrm{~T})$, differential equations considered are:

$$
\begin{array}{ll}
\frac{d I(t)}{d t}=-\left(a_{1}+b_{1} t-\rho_{1} p_{1}\right), & 0 \leq t \leq t_{1} \\
\frac{d I(t)}{d t}+\theta I(t)=-\left(a_{2}+b_{2} t-\rho_{2} p_{2}\right), & t_{1} \leq t \leq t_{2} \\
\frac{d I(t)}{d t}+\theta t I(t)=-\left(a_{3}+b_{3} t-\rho_{3} p_{3}\right), & t_{2} \leq t \leq T
\end{array}
$$

conditions initially taken as: $\mathrm{I}(0)=\mathrm{Q}, \mathrm{I}\left(\mathrm{t}_{1}\right)=\mathrm{S}_{1}, \mathrm{I}(\mathrm{T})=0$.

Respective solutions are:

$$
\begin{aligned}
I(t) & =Q-\left(a_{1} t-\rho_{1} p_{1} t+\frac{1}{2} b_{1} t^{2}\right) \\
I(t) & =\left[\begin{array}{l}
a_{2}\left(t_{1}-t\right)-\rho_{2} p_{2}\left(t_{1}-t\right)+\frac{1}{2} a_{2} \theta\left(t_{1}^{2}-t^{2}\right) \\
-\frac{1}{2} \rho_{2} p_{2} \theta\left(t_{1}^{2}-t^{2}\right)+\frac{1}{2} b_{2}\left(t_{1}^{2}-t^{2}\right)+\frac{1}{3} b_{2} \theta\left(t_{1}^{3}-t^{3}\right) \\
-a_{2} \theta t\left(t_{1}-t\right)+\rho_{2} p_{2} \theta t\left(t_{1}-t\right)-\frac{1}{2} b_{2} \theta t\left(t_{1}^{2}-t^{2}\right)
\end{array}\right] \\
+ & S_{1}\left[\begin{array}{l}
1+\theta(t) \\
\left.I\left(t_{1}-t\right)\right] \\
a_{3}(T-t)-\rho_{3} p_{3}(T-t)+\frac{1}{2} b_{3}\left(T^{2}-t^{2}\right) \\
+\frac{1}{6} a_{3} \theta\left(T^{3}-t^{3}\right)-\frac{1}{6} \rho_{3} p_{3} \theta\left(T^{3}-t^{3}\right)+\frac{1}{8} b_{3} \theta\left(T^{4}-t^{4}\right) \\
-\frac{1}{2} a_{3} \theta t^{2}(T-t)+\frac{1}{2} \rho_{3} p_{3} \theta t^{2}(T-t)-\frac{1}{4} b_{3} \theta t^{2}\left(T^{2}-t^{2}\right)
\end{array}\right]
\end{aligned}
$$

(Higher powers of $\theta$ are not considered)

$\mathrm{dQ}$ is defective items separated at time $\mu_{1}$ after screening process.

Therefore between $\mu_{1} \leq \mathrm{t} \leq \mathrm{T}$, effective inventory is:

$$
I_{1}(t)=Q(1-d)-\left(a_{1} t-\rho_{1} p_{1} t+\frac{1}{2} b_{1} t^{2}\right)
$$

Substituting $\mathrm{t}=\mathrm{t}_{1}$, in (6) gives

$$
\mathrm{Q}=\mathrm{S}_{1}+\left(\mathrm{a}_{1} \mathrm{t}_{1}-\rho_{1} \mathrm{p}_{1} \mathrm{t}_{1}+\frac{1}{2} \mathrm{~b}_{1} \mathrm{t}_{1}^{2}\right) .
$$

Under the assumption that for repairing, repairable items of amount $\mathrm{d}_{1} \%$ sent to manufacturer and before completion of cycle $\left(t_{2} \leq t \leq T\right)$, we receive back items after repairing. These repaired items before completion of cycle, are sold at original price. For sending to manufacturer and receiving back items repaired causes transportation cost. Level of stock during $\mathrm{t}_{2} \leq \mathrm{t} \leq \mathrm{T}$ is as below:

$$
\begin{aligned}
I_{3}(t) & =\left[\begin{array}{l}
a_{3}(T-t)-\rho_{3} p_{3}(T-t)+\frac{1}{2} b_{3}\left(T^{2}-t^{2}\right) \\
+\frac{1}{6} a_{3} \theta\left(T^{3}-t^{3}\right)-\frac{1}{6} \rho_{3} p_{3} \theta\left(T^{3}-t^{3}\right)+\frac{1}{8} b_{3} \theta\left(T^{4}-t^{4}\right) \\
-\frac{1}{2} a_{3} \theta t^{2}(T-t)+\frac{1}{2} \rho_{3} p_{3} \theta t^{2}(T-t)-\frac{1}{4} b_{3} \theta t^{2}\left(T^{2}-t^{2}\right)
\end{array}\right] \\
+ & d_{1} Q .
\end{aligned}
$$

Taking $\mathrm{t}=\mathrm{t}_{2}$ in equations (7) and (11), we get 


$$
\begin{aligned}
& I_{2}\left(t_{2}\right)= {\left[\begin{array}{l}
a_{2}\left(t_{1}-t_{2}\right)-\rho_{2} p_{2}\left(t_{1}-t_{2}\right)+\frac{1}{2} a_{2} \theta\left(t_{1}^{2}-t_{2}^{2}\right) \\
-\frac{1}{2} \rho_{2} p_{2} \theta\left(t_{1}^{2}-t_{2}^{2}\right)+\frac{1}{2} b_{2}\left(t_{1}^{2}-t_{2}^{2}\right)+\frac{1}{3} b_{2} \theta\left(t_{1}^{3}-t_{2}^{3}\right) \\
-a_{2} \theta t_{2}\left(t_{1}-t_{2}\right)+\rho_{2} p_{2} \theta t_{2}\left(t_{1}-t_{2}\right)-\frac{1}{2} b_{2} \theta \mu_{2}\left(t_{1}^{2}-t_{2}^{2}\right)
\end{array}\right] } \\
&+ S_{1}\left[1+\theta\left(t_{1}-t_{2}\right)\right] \\
& I_{3}\left(t_{2}\right)=\left[\begin{array}{l}
a_{3}\left(T-t_{2}\right)-\rho_{3} p_{3}\left(T-t_{2}\right)+\frac{1}{2} b_{3}\left(T^{2}-t_{2}^{2}\right) \\
+\frac{1}{6} a_{3} \theta\left(T^{3}-t_{2}^{3}\right)-\frac{1}{6} \rho_{3} p_{3} \theta\left(T^{3}-t_{2}^{3}\right)+\frac{1}{8} b_{3} \theta\left(T^{4}-t_{2}^{4}\right) \\
-\frac{1}{2} a_{3} \theta t_{2}^{2}\left(T-t_{2}\right)+\frac{1}{2} \rho_{3} p_{3} \theta \mu_{2}^{2}\left(T-t_{2}\right)-\frac{1}{4} b_{3} \theta t_{2}^{2}\left(T^{2}-t_{2}^{2}\right)
\end{array}\right] \\
&+d_{1} Q .
\end{aligned}
$$

We get from equations (12) and (13)

$$
\begin{aligned}
& S_{1}=\frac{1}{\left[1+\theta\left(t_{1}-t_{2}\right)-d_{1}\right]} \\
& {\left[\begin{array}{l}
a_{3}\left(T-t_{2}\right)-\rho_{3} p_{3}\left(T-t_{2}\right)+\frac{1}{2} b_{3}\left(T^{2}-t_{2}^{2}\right) \\
+\frac{1}{6} a_{3} \theta\left(T^{3}-t_{2}^{3}\right)-\frac{1}{6} \rho_{3} p_{3} \theta\left(T^{3}-t_{2}^{3}\right)+\frac{1}{8} b_{3} \theta\left(T^{4}-t_{2}^{4}\right) \\
-\frac{1}{2} a_{3} \theta t_{2}^{2}\left(T-t_{2}\right)+\frac{1}{2} \rho_{3} p_{3} \theta \mu_{2}^{2}\left(T-t_{2}\right) \\
-\frac{1}{4} b_{3} \theta t_{2}^{2}\left(T^{2}-t_{2}^{2}\right)-a_{2}\left(t_{1}-t_{2}\right)+\rho_{2} p_{2}\left(t_{1}-t_{2}\right) \\
-\frac{1}{2} a_{2} \theta\left(t_{1}^{2}-t_{2}^{2}\right)+\frac{1}{2} \rho_{2} p_{2} \theta\left(t_{1}^{2}-t_{2}^{2}\right) \\
-\frac{1}{2} b_{2}\left(t_{1}^{2}-t_{2}^{2}\right)-\frac{1}{3} b_{2} \theta\left(t_{1}^{3}-t_{2}^{3}\right) \\
+a_{2} \theta t_{2}\left(t_{1}-t_{2}\right)-\rho_{2} p_{2} \theta t_{2}\left(t_{1}-t_{2}\right)+\frac{1}{2} b_{2} \theta t_{2}\left(t_{1}^{2}-t_{2}^{2}\right) \\
+d_{1}\left(a_{1} t_{1}-\rho_{1} p_{1} t_{1}+\frac{1}{2} b_{1} t_{1}^{2}\right)
\end{array}\right]}
\end{aligned}
$$

We get equation (10) by substituting value of $S_{1}$ from equation (14)

$$
\begin{aligned}
Q= & \frac{1}{\left[1+\theta\left(t_{1}-t_{2}\right)-d_{1}\right]} \\
& {\left[\begin{array}{l}
a_{3}\left(T-t_{2}\right)-\rho_{3} p_{3}\left(T-t_{2}\right)+\frac{1}{2} b_{3}\left(T^{2}-t_{2}^{2}\right) \\
+\frac{1}{6} a_{3} \theta\left(T^{3}-t_{2}^{3}\right)-\frac{1}{6} \rho_{3} p_{3} \theta\left(T^{3}-t_{2}^{3}\right)+\frac{1}{8} b_{3} \theta\left(T^{4}-t_{2}^{4}\right) \\
-\frac{1}{2} a_{3} \theta t_{2}^{2}\left(T-t_{2}\right)+\frac{1}{2} \rho_{3} p_{3} \theta \mu_{2}^{2}\left(T-t_{2}\right) \\
-\frac{1}{4} b_{3} \theta t_{2}^{2}\left(T^{2}-t_{2}^{2}\right)-a_{2}\left(t_{1}-t_{2}\right)+\rho_{2} p_{2}\left(t_{1}-t_{2}\right) \\
-\frac{1}{2} a_{2} \theta\left(t_{1}^{2}-t_{2}^{2}\right)+\frac{1}{2} \rho_{2} p_{2} \theta\left(t_{1}^{2}-t_{2}^{2}\right)-\frac{1}{2} b_{2}\left(t_{1}^{2}-t_{2}^{2}\right) \\
-\frac{1}{3} b_{2} \theta\left(t_{1}^{3}-t_{2}^{3}\right)+a_{2} \theta t_{2}\left(t_{1}-t_{2}\right)-\rho_{2} p_{2} \theta t_{2}\left(t_{1}-t_{2}\right) \\
+\frac{1}{2} b_{2} \theta t_{2}\left(t_{1}^{2}-t_{2}^{2}\right)+d_{1}\left(a_{1} t_{1}-\rho_{1} p_{1} t_{1}+\frac{1}{2} b_{1} t_{1}^{2}\right)
\end{array}\right] } \\
& +\left(a_{1} t_{1}-\rho_{1} p_{1} t_{1}+\frac{1}{2} b_{1} t_{1}^{2}\right) .
\end{aligned}
$$

Using (15) in (6), gives 


$$
\begin{aligned}
& I_{1}(t)=\frac{1}{\left[1+\theta\left(t_{1}-t_{2}\right)-d_{1}\right]} \\
& {\left[\begin{array}{l}
a_{3}\left(T-t_{2}\right)-\rho_{3} p_{3}\left(T-t_{2}\right)+\frac{1}{2} b_{3}\left(T^{2}-t_{2}^{2}\right) \\
+\frac{1}{6} a_{3} \theta\left(T^{3}-t_{2}^{3}\right)-\frac{1}{6} \rho_{3} p_{3} \theta\left(T^{3}-t_{2}^{3}\right)+\frac{1}{8} b_{3} \theta\left(T^{4}-t_{2}^{4}\right) \\
-\frac{1}{2} a_{3} \theta t_{2}^{2}\left(T-t_{2}\right)+\frac{1}{2} \rho_{3} p_{3} \theta \mu_{2}^{2}\left(T-t_{2}\right) \\
-\frac{1}{4} b_{3} \theta t_{2}^{2}\left(T^{2}-t_{2}^{2}\right)-a_{2}\left(t_{1}-t_{2}\right)+\rho_{2} p_{2}\left(t_{1}-t_{2}\right) \\
-\frac{1}{2} a_{2} \theta\left(t_{1}^{2}-t_{2}^{2}\right)+\frac{1}{2} \rho_{2} p_{2} \theta\left(t_{1}^{2}-t_{2}^{2}\right)-\frac{1}{2} b_{2}\left(t_{1}^{2}-t_{2}^{2}\right) \\
-\frac{1}{3} b_{2} \theta\left(t_{1}^{3}-t_{2}^{3}\right)+a_{2} \theta t_{2}\left(t_{1}-t_{2}\right)-\rho_{2} p_{2} \theta t_{2}\left(t_{1}-t_{2}\right) \\
+\frac{1}{2} b_{2} \theta t_{2}\left(t_{1}^{2}-t_{2}^{2}\right)+d_{1}\left(a_{1} t_{1}-\rho_{1} p_{1} t_{1}+\frac{1}{2} b_{1} t_{1}^{2}\right)
\end{array}\right] } \\
&+\left(a_{1} t_{1}-\rho_{1} p_{1} t_{1}+\frac{1}{2} b_{1} t_{1}^{2}\right)-\left(a_{1} t-\rho_{1} p_{1} t+\frac{1}{2} b_{1} t^{2}\right) .
\end{aligned}
$$

Substituting (15) in (9) gives

$$
\begin{aligned}
& I_{1}(t)= \frac{(1-d)}{\left[1+\theta\left(t_{1}-t_{2}\right)-d_{1}\right]} \\
& {\left[\begin{array}{l}
a_{3}\left(T-t_{2}\right)-\rho_{3} p_{3}\left(T-t_{2}\right)+\frac{1}{2} b_{3}\left(T^{2}-t_{2}^{2}\right) \\
+\frac{1}{6} a_{3} \theta\left(T^{3}-t_{2}^{3}\right)-\frac{1}{6} \rho_{3} p_{3} \theta\left(T^{3}-t_{2}^{3}\right)+\frac{1}{8} b_{3} \theta\left(T^{4}-t_{2}^{4}\right) \\
-\frac{1}{2} a_{3} \theta t_{2}^{2}\left(T-t_{2}\right)+\frac{1}{2} \rho_{3} p_{3} \theta \mu_{2}^{2}\left(T-t_{2}\right) \\
-\frac{1}{4} b_{3} \theta t_{2}^{2}\left(T^{2}-t_{2}^{2}\right)-a_{2}\left(t_{1}-t_{2}\right) \\
+\rho_{2} p_{2}\left(t_{1}-t_{2}\right)-\frac{1}{2} a_{2} \theta\left(t_{1}^{2}-t_{2}^{2}\right)+\frac{1}{2} \rho_{2} p_{2} \theta\left(t_{1}^{2}-t_{2}^{2}\right) \\
-\frac{1}{2} b_{2}\left(t_{1}^{2}-t_{2}^{2}\right)-\frac{1}{3} b_{2} \theta\left(t_{1}^{3}-t_{2}^{3}\right)+a_{2} \theta t_{2}\left(t_{1}-t_{2}\right) \\
-\rho_{2} p_{2} \theta t_{2}\left(t_{1}-t_{2}\right)+\frac{1}{2} b_{2} \theta t_{2}\left(t_{1}^{2}-t_{2}^{2}\right) \\
+d_{1}\left(a_{1} t_{1}-\rho_{1} p_{1} t_{1}+\frac{1}{2} b_{1} t_{1}^{2}\right)
\end{array}\right] } \\
&+(1-d)\left(a_{1} t_{1}-\rho_{1} p_{1} t_{1}+\frac{1}{2} b_{1} t_{1}^{2}\right)-\left(a_{1} t-\rho_{1} p_{1} t+\frac{1}{2} b_{1} t^{2}\right)
\end{aligned}
$$

We get equation (7) by substituting value of $S_{1}$ from equation (13)

$$
\begin{gathered}
I_{2}(t)=\frac{\left[1+\theta\left(t_{1}-t\right)\right]}{\left[1+\theta\left(t_{1}-t_{2}\right)-d_{1}\right]} \\
{\left[\begin{array}{l}
a_{3}\left(T-t_{2}\right)-\rho_{3} p_{3}\left(T-t_{2}\right)+\frac{1}{2} b_{3}\left(T^{2}-t_{2}^{2}\right) \\
+\frac{1}{6} a_{3} \theta\left(T^{3}-t_{2}^{3}\right)-\frac{1}{6} \rho_{3} p_{3} \theta\left(T^{3}-t_{2}^{3}\right)+\frac{1}{8} b_{3} \theta\left(T^{4}-t_{2}^{4}\right) \\
-\frac{1}{2} a_{3} \theta t_{2}^{2}\left(T-t_{2}\right)+\frac{1}{2} \rho_{3} p_{3} \theta \mu_{2}^{2}\left(T-t_{2}\right) \\
-\frac{1}{4} b_{3} \theta t_{2}^{2}\left(T^{2}-t_{2}^{2}\right)-a_{2}\left(t_{1}-t_{2}\right)+\rho_{2} p_{2}\left(t_{1}-t_{2}\right) \\
-\frac{1}{2} a_{2} \theta\left(t_{1}^{2}-t_{2}^{2}\right)+\frac{1}{2} \rho_{2} p_{2} \theta\left(t_{1}^{2}-t_{2}^{2}\right)-\frac{1}{2} b_{2}\left(t_{1}^{2}-t_{2}^{2}\right) \\
-\frac{1}{3} b_{2} \theta\left(t_{1}^{3}-t_{2}^{3}\right)+a_{2} \theta t_{2}\left(t_{1}-t_{2}\right)-\rho_{2} p_{2} \theta t_{2}\left(t_{1}-t_{2}\right) \\
+\frac{1}{2} b_{2} \theta t_{2}\left(t_{1}^{2}-t_{2}^{2}\right)+d_{1}\left(a_{1} t_{1}-\rho_{1} p_{1} t_{1}+\frac{1}{2} b_{1} t_{1}^{2}\right)
\end{array}\right]} \\
+\left[\begin{array}{l}
a_{2}\left(t_{1}-t\right)-\rho_{2} p_{2}\left(t_{1}-t\right)+\frac{1}{2} a_{2} \theta\left(t_{1}^{2}-t^{2}\right) \\
-\frac{1}{2} \rho_{2} p_{2} \theta\left(t_{1}^{2}-t^{2}\right)+\frac{1}{2} b_{2}\left(t_{1}^{2}-t^{2}\right)+\frac{1}{3} b_{2} \theta\left(t_{1}^{3}-t^{3}\right) \\
-a_{2} \theta t\left(t_{1}-t\right)+\rho_{2} p_{2} \theta t\left(t_{1}-t\right)-\frac{1}{2} b_{2} \theta t\left(t_{1}^{2}-t^{2}\right)
\end{array}\right]
\end{gathered}
$$


Total profit $(\pi)$ consists of:

(i) Cost of ordering $(\mathrm{OC})=\mathrm{A}$

(ii) Cost of screening $(\mathrm{SrC})=\mathrm{z} Q$

(iii) Transportation cost (TC) $=\operatorname{md}_{1} \mathrm{Q}$

(iv) $\mathrm{HC}=\int_{0}^{\mathrm{T}}(\mathrm{x}+\mathrm{yt}) \mathrm{I}(\mathrm{t}) \mathrm{dt}$

$$
\begin{aligned}
= & \int_{0}^{\mu_{1}}(x+y t) I_{1}(t) d t+\int_{\mu_{1}}^{t_{1}}(x+y t) I_{1}(t) d t \\
& +\int_{t_{1}}^{t_{2}}(x+y t) I_{2}(t) d t+\int_{t_{2}}^{T}(x+y t) I_{3}(t) d t
\end{aligned}
$$

(v) $\mathrm{DC}=\mathrm{c}\left(\int_{\mathrm{t}_{1}}^{\mathrm{t}_{2}} \theta \mathrm{I}_{2}(\mathrm{t}) \mathrm{dt}+\int_{\mathrm{t}_{2}}^{\mathrm{T}} \theta \mathrm{t} \mathrm{I}_{3}(\mathrm{t}) \mathrm{dt}\right)$

(vi) $\mathrm{SR}=$ Revenue generated during the cycle

+ Revenue from imperfect quality items

+ Revenue from repaired items

$$
=\left(\begin{array}{l}
p_{1} \int_{0}^{t_{1}}\left(a_{1}+b_{1} t-\rho_{1} p_{1}\right) d t \\
+p_{2} \int_{t_{1}}^{t_{2}}\left(a_{2}+b_{2} t-\rho_{2} p_{2}\right) d t \\
+p_{3} \int_{t_{2}}^{T}\left(a_{3}+b_{3} t-\rho_{3} p_{3}\right) d t \\
+p_{d}\left(d-d_{1}\right) Q-p\left(d-d_{1}\right) Q
\end{array}\right) .
$$

(by not considering higher powers of $\theta$ )

$$
\pi=\frac{1}{\mathrm{~T}}[\mathrm{SR}-\mathrm{OC}-\mathrm{SrC}-\mathrm{TC}-\mathrm{HC}-\mathrm{DC}]
$$

Putting value in equation (25) from equations (19) to (24) provides overall unit profit. Moreover, it can be obtained in terms of $\mathrm{p}_{1}, \mathrm{p}_{2}, \mathrm{p}_{3}$ and $\mathrm{T}$ using $\mathrm{t}_{1}=\mathrm{v}_{1} \mathrm{~T}, \mathrm{t}_{2}=\mathrm{v}_{2} \mathrm{~T}$ in (25). Taking derivative with respect to $\mathrm{p}_{1}, \mathrm{p}_{2}, \mathrm{p}_{3}, \mathrm{~T}$ and equating it to zero, in equation (25), gives

$$
\frac{\partial \pi}{\partial \mathrm{p}_{1}}=0, \frac{\partial \pi}{\partial \mathrm{p}_{2}}=0, \frac{\partial \pi}{\partial \mathrm{p}_{3}}=0, \frac{\partial \pi}{\partial \mathrm{T}}=0,
$$

Moreover it has to satisfy the condition

$$
\left|\begin{array}{cccc}
\frac{\partial \pi^{2}}{\partial \mathrm{p}_{1}^{2}} & \frac{\partial \pi^{2}}{\partial \mathrm{p}_{1} \partial \mathrm{p}_{2}} & \frac{\partial \pi^{2}}{\partial \mathrm{p}_{1} \partial \mathrm{p}_{3}} & \frac{\partial \pi^{2}}{\partial \mathrm{p}_{1} \partial \mathrm{T}} \\
\frac{\partial \pi^{2}}{\partial \mathrm{p}_{2} \partial \mathrm{p}_{1}} & \frac{\partial \pi^{2}}{\partial^{2} \mathrm{p}_{2}^{2}} & \frac{\partial \pi^{2}}{\partial \mathrm{p}_{2} \partial \mathrm{p}_{3}} & \frac{\partial \pi^{2}}{\partial \mathrm{p}_{2} \partial \mathrm{T}} \\
\frac{\partial \pi^{2}}{\partial \mathrm{p}_{3} \partial \mathrm{p}_{1}} & \frac{\partial \pi^{2}}{\partial \mathrm{p}_{3} \partial \mathrm{p}_{2}} & \frac{\partial \pi^{2}}{\partial^{2} \mathrm{p}_{3}^{2}} & \frac{\partial \pi^{2}}{\partial \mathrm{p}_{3} \partial \mathrm{T}} \\
\frac{\partial \pi^{2}}{\partial \mathrm{T} \partial \mathrm{p}_{1}} & \frac{\partial \pi^{2}}{\partial \mathrm{T} \partial \mathrm{p}_{2}} & \frac{\partial \pi^{2}}{\partial \mathrm{T} \partial \mathrm{p}_{3}} & \frac{\partial \pi^{2}}{\partial^{2} \mathrm{~T}^{2}}
\end{array}\right|>0 .
$$

\section{NUMERICAL EXAMPLE}

Considering $A=$ Rs.100, $a_{1}=500, a_{2}=490$, $\mathrm{a}_{3}=480, \mathrm{~b}_{1}=0.04, \mathrm{~b}_{2}=0.05, \mathrm{~b}_{3}=0.06, \mathrm{c}=25, \mathrm{p}_{\mathrm{d}}=15, \mathrm{~d}=$ $0.05, \mathrm{~d}_{1}=0.03, \mathrm{z}=0.40, \mathrm{~m}=70, \theta=0.05, \mathrm{x}=5, \mathrm{y}$ $=0.05, \rho_{1}=4, \rho_{2}=4.5, \rho_{3}=5, v_{1}=0.30, v_{2}=0.50$, in suitable units. Optimal values are: $\mathrm{p}_{1} *=62.4150$, $\mathrm{p}_{2} *=56.6567, \quad \mathrm{p}_{3}^{*}=50.5989, \quad \mathrm{~T} *=0.3921$, Profit $*=11750.6414$ and $Q^{*}=92.6572$.

Equation (27) is also satisfied. Graphs for prices and profit are also shown below. 


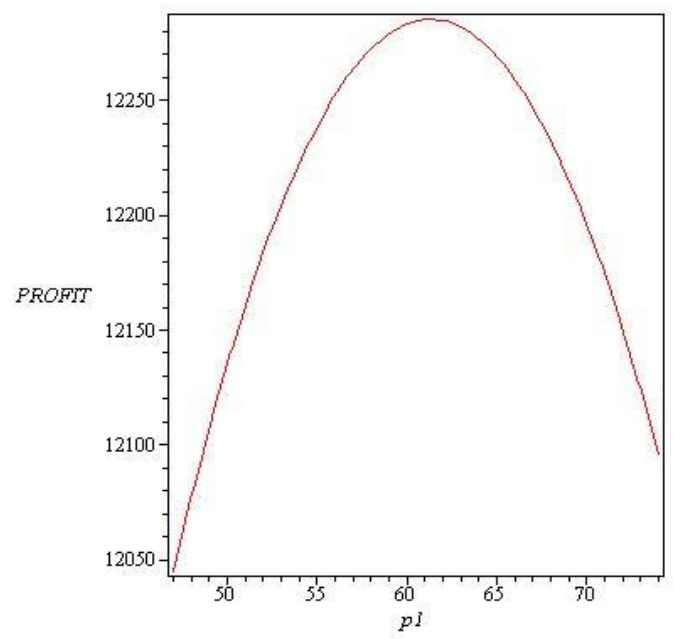

Fig-2: $p_{1}$ and Profit

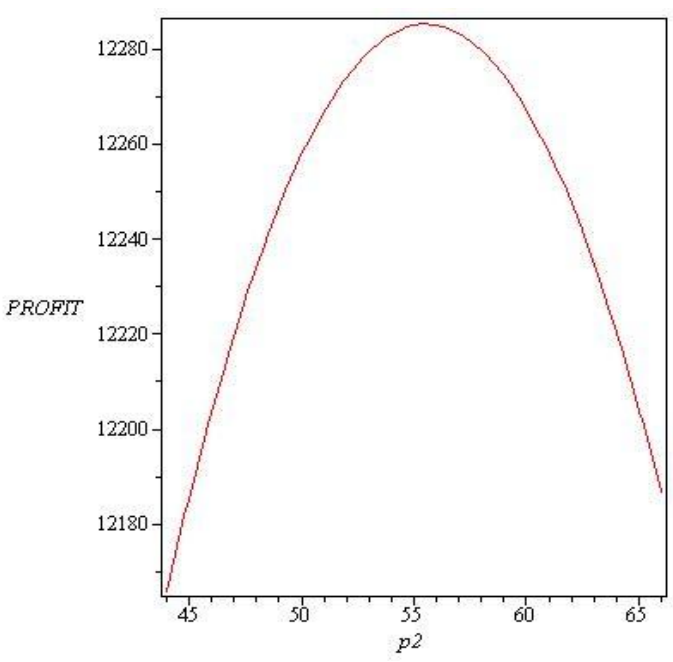

Fig-3: $p_{2}$ and Profit

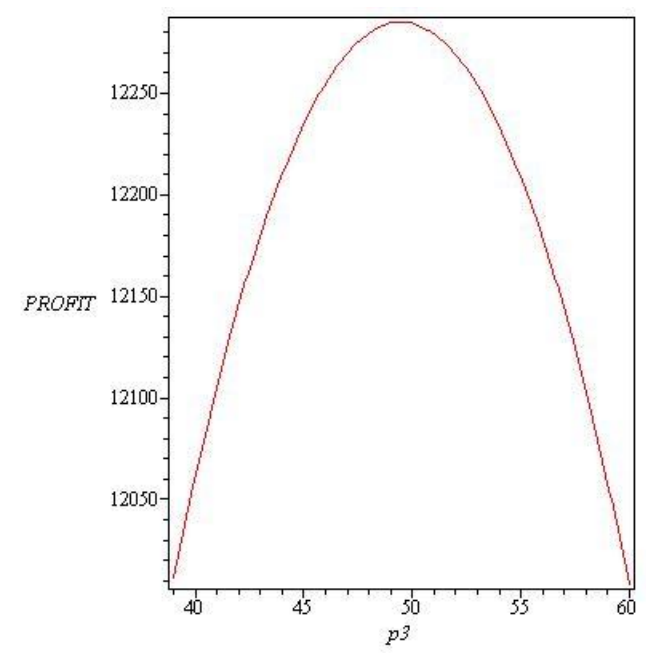

Fig-4: $p_{3}$ and Profit

\section{POST-OPTIMALITY ANALYSIS}

Study of one parameter at a time, table below gives post-optimality computations. 
D.M. Patel \& R.D. Patel., Sch J Phys Math Stat, May, 2021; 8(5): 96-104

Table-1: Post-optimality Analysis

\begin{tabular}{|c|c|c|c|c|c|c|c|}
\hline Parameter & $\%$ & $\mathrm{~T}$ & $\mathrm{p}_{1}$ & $\mathrm{p}_{2}$ & $\mathrm{p}_{3}$ & Profit & Q \\
\hline \multirow{4}{*}{$a_{1}$} & $+20 \%$ & 0.3886 & 74.9173 & 56.7822 & 50.7212 & 13690.0944 & 97.4917 \\
\hline & $+10 \%$ & 0.3903 & 68.6661 & 56.7195 & 50.6600 & 12674.4407 & 95.0750 \\
\hline & $-10 \%$ & 0.3938 & 56.1638 & 56.5940 & 50.5378 & 10918.6967 & 90.1902 \\
\hline & $-20 \%$ & 0.3956 & 49.9126 & 56.5314 & 50.4767 & 10178.6067 & 87.7206 \\
\hline \multirow{4}{*}{$a_{2}$} & $+20 \%$ & 0.3857 & 62.3259 & 67.5378 & 50.5845 & 12881.2137 & 94.9846 \\
\hline & $+10 \%$ & 0.3889 & 62.3704 & 62.0972 & 50.5916 & 12289.2306 & 93.8370 \\
\hline & $-10 \%$ & 0.3954 & 62.4595 & 51.2163 & 50.6063 & 11265.4469 & 91.4686 \\
\hline & $-20 \%$ & 0.3988 & 62.5042 & 45.7760 & 50.6139 & 10833.6480 & 90.2695 \\
\hline \multirow{4}{*}{$a_{3}$} & $+20 \%$ & 0.3647 & 62.1906 & 56.6243 & 60.1384 & 14161.9612 & 95.1199 \\
\hline & $+10 \%$ & 0.3776 & 62.3024 & 56.6397 & 55.3670 & 12898.3408 & 93.8598 \\
\hline & $-10 \%$ & 0.4083 & 62.5284 & 56.6758 & 45.8347 & 10718.9489 & 91.4739 \\
\hline & $-20 \%$ & 0.4269 & 62.6430 & 56.6974 & 41.0754 & 9803.3686 & 90.3936 \\
\hline \multirow{4}{*}{$\mathrm{X}$} & $+20 \%$ & 0.3613 & 62.4350 & 56.6969 & 50.6710 & 11706.2453 & 85.2680 \\
\hline & $+10 \%$ & 0.3757 & 62.4252 & 56.6773 & 50.6357 & 11727.9807 & 88.7222 \\
\hline & $-10 \%$ & 0.4107 & 62.4041 & 56.6352 & 50.5604 & 11774.3537 & 97.1223 \\
\hline & $-20 \%$ & 0.4323 & 62.3926 & 56.6125 & 50.5200 & 11799.2753 & 102.3096 \\
\hline \multirow{4}{*}{$\theta$} & $+20 \%$ & 0.3904 & 62.4134 & 56.6609 & 50.6045 & 11747.9225 & 92.3013 \\
\hline & $+10 \%$ & 0.3912 & 62.4142 & 56.6588 & 50.6017 & 11749.2812 & 92.4675 \\
\hline & $-10 \%$ & 0.3929 & 62.4157 & 56.6547 & 50.5960 & 11752.0031 & 92.8231 \\
\hline & $-20 \%$ & 0.3938 & 62.4165 & 56.6526 & 50.5932 & 11753.3664 & 93.0123 \\
\hline \multirow{4}{*}{ A } & $+20 \%$ & 0.4291 & 62.4326 & 56.6982 & 50.6784 & 11701.9334 & 101.3246 \\
\hline & $+10 \%$ & 0.4110 & 62.4240 & 56.6779 & 50.6395 & 11725.7389 & 97.0861 \\
\hline & $-10 \%$ & 0.3721 & 62.4055 & 56.6344 & 50.5562 & 11776.8103 & 87.9667 \\
\hline & $-20 \%$ & 0.3511 & 62.3954 & 56.6109 & 50.5111 & 11804.4634 & 83.0382 \\
\hline \multirow{4}{*}{$\rho_{1}$} & $+20 \%$ & 0.3919 & 52.2312 & 56.5513 & 50.4929 & 11020.1836 & 92.6280 \\
\hline & $+10 \%$ & 0.3920 & 56.8601 & 56.5992 & 50.5411 & 11352.1816 & 92.6491 \\
\hline & $-10 \%$ & 0.3922 & 69.2047 & 56.7270 & 50.6695 & 12237.7246 & 92.6480 \\
\hline & $-20 \%$ & 0.3924 & 77.6925 & 56.8149 & 50.7579 & 12846.6777 & 92.6385 \\
\hline \multirow{4}{*}{$\rho_{2}$} & $+20 \%$ & 0.3923 & 62.4186 & 47.5828 & 50.5994 & 11306.8780 & 92.5458 \\
\hline & $+10 \%$ & 0.3922 & 62.4168 & 51.7073 & 50.5991 & 11508.5486 & 92.6016 \\
\hline & $-10 \%$ & 0.3920 & 62.4131 & 62.7060 & 50.5986 & 12046.6306 & 92.7129 \\
\hline & $-20 \%$ & 0.3918 & 62.4113 & 70.2678 & 50.5983 & 12416.7270 & 92.7447 \\
\hline \multirow{4}{*}{$\rho_{3}$} & $+20 \%$ & 0.3937 & 62.4272 & 56.6586 & 42.6021 & 10793.9478 & 92.5121 \\
\hline & $+10 \%$ & 0.3929 & 62.4211 & 56.6577 & 46.4368 & 11228.6539 & 92.3682 \\
\hline & $-10 \%$ & 0.3913 & 62.4089 & 56.6558 & 55.9306 & 12389.0033 & 92.7277 \\
\hline & $-20 \%$ & 0.3905 & 62.4028 & 56.6549 & 62.5958 & 13187.3791 & 92.7966 \\
\hline
\end{tabular}

Calculations of Table 1 shows that increase or decrease in value of profit and order quantity occur, when parameters ' $a_{1}$ ', ' $a_{2}$ ', ' $a_{3}$ ' increase/decrease.

Also when parameters ' $\theta$ ' and ' $x$ ' increase/ decrease then total profit and order quantity decrease/ increase. Moreover, when increase/decrease in parameters ' $A$ ' and ' $\rho_{1}$ ' occurs, then profit also shows decrease/increase and order quantity shows increase/ decrease. Also when parameters ' $\rho_{2}$ ' and ' $\rho_{3}$ ' increase/ decrease then profit and order quantity decrease/ increase.

\section{SPECIAL CASE}

Taking $\mathrm{d}=0, \mathrm{~d}_{1}=0, \mathrm{p}_{\mathrm{d}}=0, \mathrm{z}=0, \mathrm{~m}=0$ gives

$\mathrm{T}^{*}=0.3908, \mathrm{p}_{1}^{*}=62.6469, \mathrm{p}_{2}^{*}=54.8515, \mathrm{p}_{3}^{*}=$ 48.8029, Profit $*=$ Rs. 12606.6509 .

The result corresponds with result obtained by Patel and Patel [2021].

\section{CONCLUSION}

Price and time dependent demand for imperfect quality and repairable items under three tire pricing inventory model is developed. For major parameters, post-optimality analysis is done. There will be variations in profit and order quantity with variations in parameter values.

\section{REFERENCES}

1. Aggarwal, S.P. and Goel, V.P. (1984): Order level inventory system with demand pattern for deteriorating items; Eco. Comp. Econ. Cybernet, Stud. Res., Vol. 3, pp. 57-69.

2. Alfares, H.(2007): Inventory model with stock level dependent demand rate and variable holding cost; International J. Production Economics, Vol. 108, pp. 259-265.

3. Cheng, T.C.E. (1991): An economic quantity model with demand dependent unit production cost 
and imperfect production process; IIE Transactions, Vol. 23, pp. 23-28.

4. Covert, R.P. and Philip, G.C. (1973): An EOQ model for items with Weibull distribution deterioration; American Institute of Industrial Engineering Transactions, Vol. 5, pp. 323-328.

5. Ghare, P.N. and Schrader, G.F. (1963): A model for exponentially decaying inventories; J. Indus. Engg., Vol. 15, pp. 238-243.

6. Gothi, U.B., Joshi, M. and Parmar, K. (2017): An inventory model of repairable items with exponential deterioration and linear demand rate; IOSR Journal of Mathematics, Vol. 13, No. 3, pp. 75-82.

7. Goyal, S.K. and Barron, L.E.C. (2002): Note on economic production quantity model for item with imperfect quality - a practical approach; Int. J. Prod. Eco., Vol. 77, pp. 85-87.

8. Hayek, P.A., Salameh, M.K., 2001. Production lot sizing with the reworking of imperfect quality items produced. Production Planning and Control 12, 584-590.

9. Hsu, J.T. and Hsu, L.F. (2012): A note on optimal inventory model for items with imperfect quality and shortage backlogging; Int. J. Ind. Engg. Comp., Vol. 3, pp. 939-948.

10. Jaber, M.Y., Goyal, S.K. and Imran, M. (2008): Economic production quantity model for items with imperfect quality subject to learning effects; International J. Prod. Eco., Vol. 115, pp. 143-150.

11. Lee, H.L. and Rosenblatt, M.J. (1985): Optimal inspection and ordering policies for products with imperfect quality; IIE Transactions, Vol. 17, No. 3, pp. 284-289.

12. Mabini, M.C., Pintelon, L.M., \& Gelders, L.F. (1992): EOQ type formulation for controlling repairable inventories; International Journal of Production Economics, Vol. 28, pp. 21-33.

13. Mathew, R.J. (2013): Perishable inventory model having mixture of Weibull lifetime and demand as function of both selling price and time; International $J$. of Scientific and Research Publication, Vol. 3(7), pp. 1-8.

14. Mukhopadhyay, R.N., Mukherjee, R.N. and Chaudhary, K.S. (2004): Joint pricing and ordering policy for deteriorating inventory; Computers and Industrial Engineering, Vol. 47, pp. 339-349.

15. Naik, B.T. and Patel, R. (2017): Inventory model with different deterioration rates for imperfect quality items under price and time dependent demand; Global Journal of Pure and Applied Mathematics, Vol. 13, No. 9, pp. 6907-6918.
16. Naik, B.T. and Patel, R. (2018): Imperfect Quality And Repairable Items Inventory Model With Different Deterioration Rates Under Price And Time Dependent Demand; International Journal Of Engineering Research And Development, Vol. 14, No. 7, pp. 41-48.

17. Papachristos, S. and Konstantaras, I. (2006): Economic ordering quantity models for items with imperfect quality; International J. Prod. Eco., Vol. 100(1), pp. 148-154.

18. Patel, D. M. and Patel, R.D. (2021) : Deteriorating items inventory model under different deterioration with three tired prices and time dependent demand; International $\mathbf{J}$. of Science Technology and Management, Vol. 10, No. 4, pp. 148-156.

19. Patel, R. and Sheikh, S.R. (2015): Inventory Model with Different Deterioration Rates under Linear Demand and Time Varying Holding Cost; International J. Mathematics and Statistics Invention, Vol. 3, No. 6, pp. 36-42.

20. Salameh, M.K. and Jaber, M.Y. (2000): Economic production quantity model for items with imperfect quality; J. Production Eco., Vol. 64, pp. 59-64.

21. Schrady, D.A. (1967): A deterministic Inventory model for repairable items; Naval Research Logistics Quarerly, Vol. 14, No. 3, pp. 391-398.

22. Schwaller, R.L., 1988: EOQ under inspection costs. Production and Inventory Management 29, 22-24.

23. Shukla, H.S., Tripathi, R.P. and Sang, N. (2016): Economic production quantity model for defective items under deterioration; Uncertain Supply Chain Management, Vol. 4, pp. 221-232.

24. Taleizadeh, A.A., Kalantari, S.S.and CárdenasBarrón, L.E. (2015): Determining optimal price, replenishment lot size and number of shipments for an EPQ model with rework and multiple shipments; J. of Industrial and Management Optimization, Vol. 11, pp. 1059-1071.

25. Teng, J.T. and Chang, H.T. (2005): Economic production quantity model for deteriorating items with price and stock dependent demand; Computers and Oper. Res., Vol. 32, pp. 279-308.

26. Tsao, Y.C., Zhang, Q., Fang, H.P. and Lee, P.L. (2017): Two tired pricing and ordering for noninstantaneous deteriorating items under trade credits: Oper. Res. Int. J., DOI 10.1007/s12351017-0306-9.

27. Yadav, R.K. and Kumar, R. (2014): An inventory model for repairable items with linear demand; IOSR Journal of Mathematics, Vol. 10, No. 6, pp. $1-6$. 